This item was submitted to Loughborough's Research Repository by the author.

Items in Figshare are protected by copyright, with all rights reserved, unless otherwise indicated.

\title{
Propagation modes of surface discharge plasma in a metallized polymer film capacitor
}

PLEASE CITE THE PUBLISHED VERSION

PUBLISHER

(c) IEEE

VERSION

VoR (Version of Record)

\section{LICENCE}

CC BY-NC-ND 4.0

\section{REPOSITORY RECORD}

Lee, Yuen-Pen, and Michael G. Kong. 2019. "Propagation Modes of Surface Discharge Plasma in a Metallized Polymer Film Capacitor". figshare. https://hdl.handle.net/2134/5193. 
This item was submitted to Loughborough's Institutional Repository (https://dspace.lboro.ac.uk/) by the author and is made available under the following Creative Commons Licence conditions.

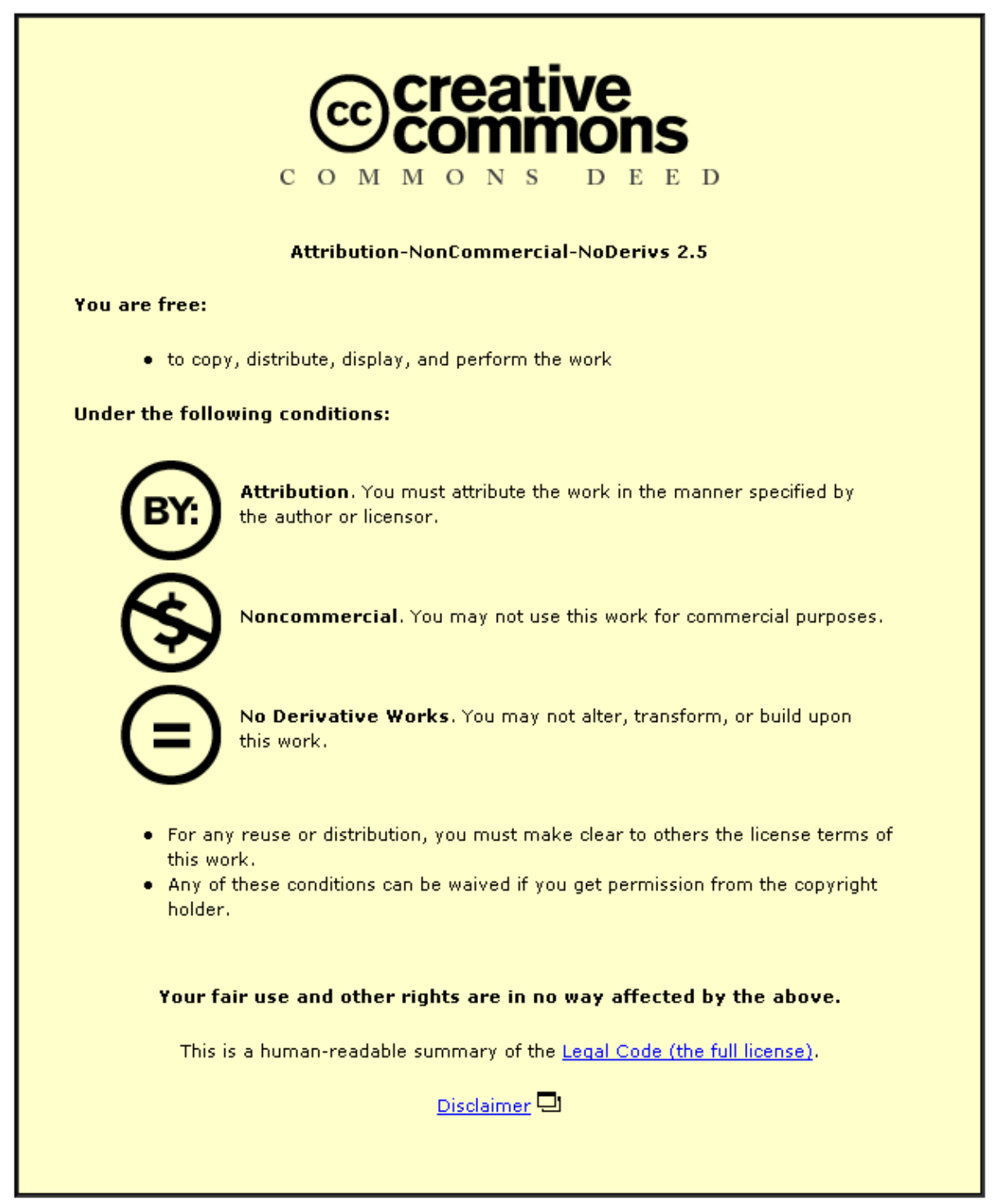

For the full text of this licence, please go to: http://creativecommons.org/licenses/by-nc-nd/2.5/ 
2001 Annual Report Conference on Electrical Insulation and Dielectric Phenomena

\title{
Propagation Modes of Surface Discharge Plasma In a Metallized Polymer Film Capacitor
}

\author{
Y P Lee and M G Kong \\ Department of Electronic and Electrical Engineering \\ Loughborough University, Leicestershire LE11 3TU, UK
}

\begin{abstract}
Surface breakdowns and their associated discharges are an important failure mechanism of metallized polymer film capacitors. To assess propagation of such discharges on the electrode coating surface and hence the impact on capacitor performance, we have recently developed a surface plasma model under non-equilibrium conditions. Through the identification of an electrical field mechanism and a temperature gradient mechanism, we have established a parametric range within which these surface plasmas are unlikely to elongate. Theoretical models of surface discharges are useful for a quantitative understanding of failure mechanisms in film capacitors. In this contribution, we extend our work further to study the dynamics of plasma propagation. Two propagation modes are identified, namely the elongation and extinction modes, and they are numerically explored over a very wide parametric range. Detailed features of plasma propagation provide important insights into their impact on capacitor performance. These results are also useful to aid optimization of capacitor designs through capacitor electrode architecture and insulation clearance.
\end{abstract}

\section{Introduction:}

There has been significant progress in the technology of metallised polymeric film capacitors over the past 20 years, fuelled by their widespread applications in power systems [1][2]. One of the main drivers in the field is higher energy density with increasingly compact designs. This highlights the need to gain a thorough understanding of propagation processes of surface discharge plasmas developed within film capacitors under higher and higher electric stress and different combinations of key system parameters.

In this paper, we study propagation modes of surface discharge on the electrode surface using a recently developed theoretical model[3]. We will extend this previous work further to study the dynamics of plasma propagation processes. Numerical examples will then be used to provide insights into the impact of surface discharge propagation processes in film capacitors under different parametric ranges.

\section{Basic model of surface discharge:}

A thorough knowledge of surface discharges requires an understanding of their ignition and that of their subsequent evolution. It is known that surface discharges in film capacitors are usually triggered by localized surface field intensification, usually caused by surface defects resulting from various manufacturing processes. To ensure the reliability and lifetime of capacitors, it is very important that these surface discharges do not elongate and develop into catastrophic flashover arcs to fail the capacitors. Therefore propagation processes of surface discharge plasma under different conditions are an important factor to influence capacitor performance.

Once a breakdown discharge is ignited from a surface defect site, it may propagate on electrode surface of a film capacitor. We have developed two simplified analytical models to study propagation and elongation of surface breakdown plasmas in metallized film capacitors under, respectively, thermal equilibrium conditions and thermally non-equilibrium conditions[3]. A surface field gradient mechanism was used to characterize propagation of cold surface plasmas whereas a temperature gradient mechanism was proposed to capture the essential features of hot surface plasmas when propagating and elongating on electrode surface. Through a numerical assessment of the worst scenario[3], it has been shown that while surface breakdown discharges may elongate they are unlikely to evolve into significant flashover arcs.

It should be mentioned that the above conclusion was reached for the square metallization pattern of figure 1 [4], for which surface resistance is known and the performance of relevant practical film capacitors is also known. For film capacitors of radically different metallization patterns, their surface resistance can be much greater than that assumed for figure 1 and their induced surface field intensification may also be significant. As a result, the impact of surface breakdown discharges needs to be reassessed for these different film capacitor parameters.

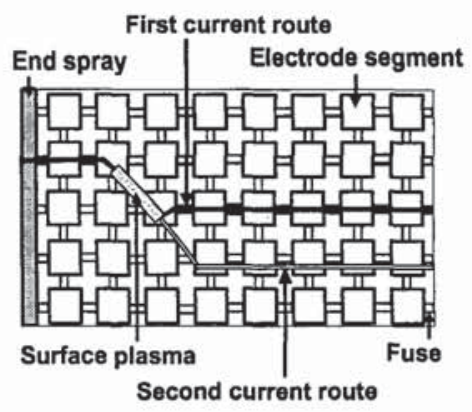

Figure 1. A simplified model films capacitor with its patterned electrode coating and side margin. 
It is known that breakdown discharges in film capacitors can be much hotter than the plasma containing capacitor structure. Furthermore the plasma temperature also changes, as the surface plasma propagates on the electrode surface and interacts thermally with its surrounding structure. Suppose the discharge plasma is not in thermal equilibrium with its surrounding capacitor structure. Its propagation on the electrode surface will be predominately controlled by the temperature gradient mechanism.

Surface discharge plasmas are usually ignited by surface electric field and subsequently sustained through the surface current. Without heat exchange with its surrounding space, the plasma is heated up through its own ohmic losses, $i_{p}{ }^{2} R_{p}$, and as such its temperature rises. However as it interacts thermally with the capacitor structure, its temperature reduces in the form of heat dissipation and radiation into the surrounding capacitor space. Therefore there are two competing processes for plasma temperature to change. If the net temperature change is negative, the plasma becomes increasingly resistive and as such increasingly unlikely to elongate. On the other hand if the plasma undergoes a positive net temperature change, it becomes increasingly hotter and as such less resistive. In general this does not necessarily lead to plasma elongation. However when the plasma resistance reduces to below the surface resistance of its surrounding electrode area, the discharge plasma begins to elongate driven by the greater electric field outside its column under the surface field gradient mechanism[3]. For cases where the plasma resistance remains larger than the electrode surface resistance throughout the timescale of interest, it is possible for the plasma front (its moving root) to sufficiently heat up the insulating air nearby for the latter to ionize. This air ionization results in a second discharge plasma, which merges with the initial plasma to become a new plasma column of greater length. In other words, this ionization mechanism can lead to an effective plasma elongation. Finally when there is no net temperature change, the plasma resistance remains unchanged and this represents the critical condition under which plasma elongation is not thermally encouraged.

As one-dimensional approximation, the surface discharge is assumed to be a uniform cylindrical column having a length of $L_{p} . L$ is the overall length of the current path. The ratio of temperature change is given by [3]

$f(T) \equiv \frac{\left|\Delta T_{\text {rise }}\right|}{\left|\Delta T_{\text {drop }}\right|}=\frac{\left(1-L_{p} / L\right)^{2}}{\left.\mid 1+\left(r_{p} / r_{s}-1\right) L_{p} / L\right]^{2}} \Theta(T)$

where

$\Theta(T)=\frac{4}{\pi^{2}} \frac{d_{p} L_{p}}{d_{c}^{2}} \frac{E_{0}^{2} r_{p} / r_{s}^{2}}{c_{p} \rho_{m p} \alpha_{s}\left(T-T_{a m b}\right)}$

$r_{p}$ and $r_{s}$ are respectively the plasma resistance and the total resistance of non-plasma section per unit length. $E_{o}$ is the average surface electric field across the surface electrode layer. $c_{p}$ and $\rho_{m p}$ are the specific heat and density of the plasma column respectively. $\alpha_{\mathrm{s}}$ is the thermal diffusivity of the electrode material. $T$ and $T_{p}$ are the ambient temperature of the capacitor unit and the plasma temperature respectively. $d_{p}$ and $d_{c}$ are the plasma diameter and the thickness of the capacitor pack.

\section{Propagation processes of surface discharge:}

For comparison, the surface discharge propagation processes for a typical practical film capacitor is used as a reference to other system parameters. To illustrate the surface discharge propagation processes under thermally non-equilibrium conditions, we considered a film capacitor with $r_{s}=200 \Omega / \mathrm{cm}$ and $T_{a m b}=298 \mathrm{~K}$. For practical multiple layer film capacitors, $d_{c}=2-5 \mathrm{~cm}$ and so we choose $d_{c}=2.5 \mathrm{~cm}$ for all cases discussed here. We now assume that the surface discharge is an air plasma with a diameter of $d_{p}=2 \mu \mathrm{m}$ and employ eq. (2) to identify the parametric condition under which the air plasma may undergo a net temperature rise. Specific heat of the air plasma is obtained from [5] as a function of temperature at one atmospheric pressure. Similarly electrical conductivity of the air plasma and its density are based on data in [6] and [7] respectively. For the electrode surface, thermal diffusivity of aluminium is shown in [8]. It is important to mention that we choose deliberately very high temperature for surface plasmas in an attempt to assess the worst scenario in which the film capacitor is impacted on by the most severe and hottest possible plasma.

One implication is that the use of eq.(2) and data in [5][8] permits an indirect description of the dynamics of surface discharge by assuming that the plasma column will become longer if the net temperature rise is positive. Surface electric field may be estimated based on an equivalent circuit model developed recently for film capacitors[4]. It has been shown that for AC capacitors at $15 \mathrm{kHz}$ or below, the rms value of surface electric field is at most $20 \mathrm{~V} / \mathrm{cm}$ [4]. Higher surface electric field can be achieved at higher frequencies, but the very short period of these high frequency signals is such that they may not be able to support a surface discharge throughout the entire duration of its development. In other words, surface discharges are likely to experience an effective electric field that is much less than predicted for frequencies much greater than $15 \mathrm{kHz}$. To this end, we assume that the surface electric field is a constant at $20 \mathrm{~V} / \mathrm{cm}$.

Based on the parameters chosen above and eqs.(1) and (2), the normalized temperature rise of the plasma column is plotted in figure 2 as a function of its initial temperature using data in [5]-[8]. Suppose a surface discharge is initially $10^{-8} \mathrm{~L}$ long (1.5nm on a $15 \mathrm{~cm}$ wide capacitor film) at about $5000 \mathrm{~K}$, corresponding to a plasma operation point at point $A$ 
in figure 2. Since there is a positive net temperature rise at point $A$, the plasma temperature goes up subsequently and its operation point moves along the curve towards right to the higher temperature region in figure 2. As the plasma becomes hotter and hotter, it may start to elongate through either an electric field gradient at its boundary with the nonplasma section or an amalgamation with an additional ionized air induced around its moving root. In figure 2, this corresponds to the plasma operation point moves to a higher curve having a greater $L p / L$ ratio (thus longer plasma length), for instance the $L_{p} / L=1 \times 10^{-7}$ curve. If the new operating point of the discharge plasma still has a positive net temperature rise, the plasma temperature continues to increase until further elongation processes move the plasma to an operation point with a zero net temperature rise such as point $C$ in figure 2 . With a surface electric field at $20 \mathrm{~V} / \mathrm{cm}$, the plasma column at point $C$ in figure 2 would be maintained around $6750 \mathrm{~K}$ and $0.01 \mathrm{~L}(1.5 \mathrm{~mm}$ on a $15 \mathrm{~cm}$ wide capacitor film). In practice, a constant surface field can very rarely be maintained forever. When and as the surface field reduces, the plasma will be gradually absorbed thermally into its surrounding structure and eventually extinguished.

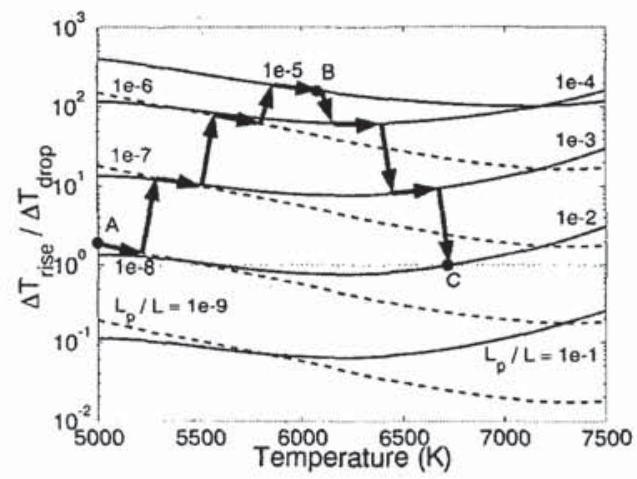

Figure 2. Normalized temperature rise of air plasma as a function of its initial temperature at different initial plasma lengths.

For surface discharges with different initial lengths from $10^{-8} \mathrm{~L}$, they are likely to undergo a similar process to the $A \rightarrow$ $B \rightarrow C$ route in figure 2 provided they experience initially a net temperature rise. Specifically a discharge plasma is likely to undergo either a field gradient induced elongation or a gas ionization induced elongation such that it settles to an equilibrium point like point $C$ in figure 2 before being absorbed thermally. For the cases where the positive net temperature rise increases as $L_{p} / L$ increases, we identify this propagation mode as the elongation mode. The surface discharge processes for the $A \rightarrow B$ route in figure 2 undergo are characteristic of an elongation mode. If the surface discharge propagation processes still on elongation mode after point $B$, there is a possibility for the surface discharge to evolve into a significant flashover arc.
For cases where the positive net temperature rise decreases as the $L_{p} / L$ ratio increases, we refer to this propagation mode as the extinction mode. The surface discharge processes for the $B \rightarrow C$ route in figure 2 represent an extinction mode. If the surface discharge propagation process are in the extinction mode and moves to an operation point with a zero net temperature rise, such as point $C$ in figure 2 , and at $L_{p} / L$ $<1$, it is highly probable that the discharge plasma will extinguish.

For surface plasmas with a negative net temperature rise initially, such as those on $L_{p} / L>0.01$ or $L_{p} / L<10^{-8}$ curves in figure 2 , they become increasingly colder and less resistive. Thus it is unlikely for them to elongate subsequently. Instead they shrink in size gradually and extinguish eventually. From the above discussion of figure 2 , it appears that there are always mechanisms for surface discharges of varying initial conditions to extinguish at an eventual length much smaller than the width of the capacitor film. Hence surface breakdowns and their discharge plasmas are not very likely to evolve into significant flashover arcs for the system parameters used in figure 2 .

At a greater surface electric field, for instance $200 \mathrm{~V} / \mathrm{cm}$, one magnitude greater than that used in figure 2 , the normalized temperature rise of the plasma column is plotted in figure 3 . The other parameters are identical to the parameters used in figure 2. Comparing figure 3 with figure 2, the surface discharges in figure 3 are likely to undergo a similar process to the $A \rightarrow B \rightarrow C$ route in figure 2, apart from the magnitude of the temperature rise in figure 3 was increased by two magnitudes with respect to the magnitude in figure 2 . Eq. (2) suggests that the normalised temperature rise is linearly proportional to the square of $E_{o}$. It is interesting to note that the plasma column at the operation point $C$ $(0.1 L, L)$ still has a positive net temperature rise. Hence at $200 \mathrm{~V} / \mathrm{cm}$, the surface discharges have a much greater tendency to evolve into a significant flashover arc.

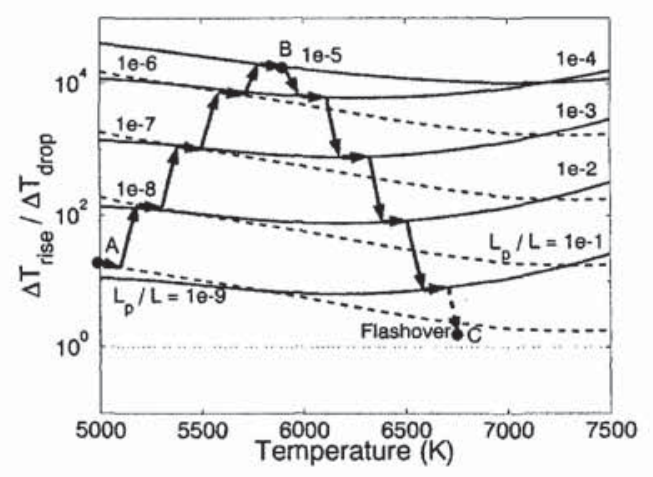

Figure 3. Normalized temperature rise of air plasma as a function of its initial temperature at $\mathrm{E}_{\mathrm{o}}$ increase by one magnitude. 
Equally important is the effect of the plasma diameter on plasma propagation processes. To illustrate this, we choose a plasma diameter one magnitude greater than that used in figure 2. The normalized temperature rise of the plasma column is re-calculated and plotted in figure 4 . The other parameters are identical to the parameters used in figure 2. Comparing figure 4 with figure 2 , the elongation mode $(A \rightarrow$ $B$ route in figure 4) covers a greater range of the plasma length with the shortest initial length at $10^{-7} \mathrm{~L}$. Towards point $C$ in figure $4\left(10^{-1} L\right)$, the surface plasma still has a positive temperature rise. Therefore at a greater plasma diameter, the surface discharge has a greater tendency to evolve into a significant flashover arc.

At a stronger surface electric field or a larger plasma diameter, the surface discharge propagation processes are different compared with the reference discharge propagation in figure 2 with a momentary tendency to evolve into a significant flashover arc. The numerical examples show that for surface discharge to extinguish at a shorter length without evolving into flashover arcs, both surface propagation process modes need to be short. If the initial discharge starts at the elongation mode, the discharge propagation needs to progress towards the extinction mode before the discharge column extends to the width of the capacitor film. It is also interesting to note that the plasma diameter used in figure 4 is much higher than that typical for film capacitors. However the surface electric field used in figure 3 is possible as the film capacitor design approaching higher energy density with increasingly compact designs.

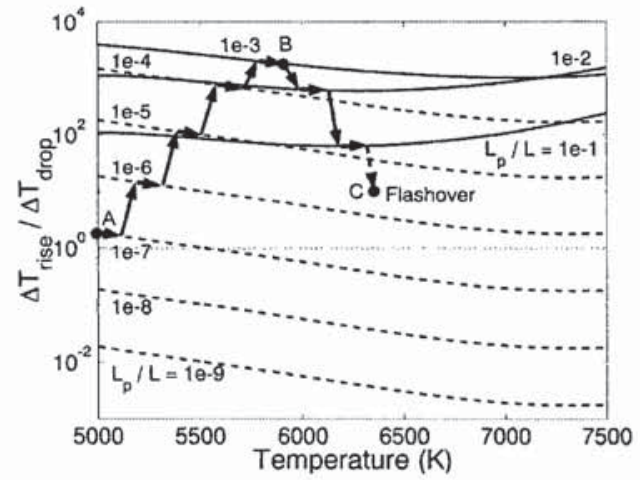

Figure 4. Normalized temperature rise of air plasma as a function of its initial temperature at $d_{p}$ increase by one magnitude.

\section{Conclusion:}

We have developed a theoretical model to study propagation processes of surface breakdown discharges in metallized polymeric film capacitors under thermally non-equilibrium conditions. A temperature gradient mechanism was used to capture the essential features of hot surface plasma when propagation on electrode surface. The numerical examples shown that the propagation processes of surface discharges can be classified into two modes, namely the elongation and extinction modes. From our study of propagation processes, it has been shown that surface breakdown discharges propagate in the elongation mode first then progress into the extinguish mode. To avoid the breakdown discharge elongating into a significant flashover arc and causing less impact on capacitor performance, both surface propagation process modes need to be short and progress toward extinction mode with a zero net temperature rise before the discharge column extends to the width of the capacitor film.

\section{Acknowledgement:}

YPL acknowledges financial support from ABB Power T \& D, Capacitor Division, UK, and Loughborough University.

\section{Reference:}

[1] D. G. Shaw, S. W. Cichanowski, and A. Yializis, "A changing capacitor technology - failure mechanism and design innovations", IEEE Trans Electrical Insulation, vol.16, pp. $399-413,1981$.

[2] C. W. Reed and S. W. Chichanowski, "The fundamentals of ageing in HV power film capacitors", IEEE Trans Dielectric \& Electrical Insulation, vol.1, pp. $904-922,1994$.

[3] M. G. Kong and Y. P. Lee, "Surface flashover dynamics in metallized polymer film capacitors", presented at the 2000 IEEE International Conference on Dielectrics and Electrical Insulation Phenomena held in Victoria, BC, Canada, October 2000.

[4] Y. P. Lee, M. G. Kong, M. R. Dunn, and D YoungCannon, "Temporal evolution of electric field distribution and energy dissipation in a metallized polymer film capacitor under dc, ac, or nonsinusoidal voltage stresses", presented at the 2000 IEEE International Conference on Dielectrics and Electrical Insulation Phenomena held in Victoria, BC, Canada, October 2000.

[5] R. N. Gupita et al, Calculations and curve fits of thermodynamic and transport properties for equilibrium air to $30000 K$, NASA Reference Publication 1260 , 1991.

[6] S. V. Dresvin, Physics and technology of lowtemperature plasmas, The Iowa State University Press, Ames, 1977.

[7] H. Kroepelin et al, Thermodynamic diagrams for high temperature plasmas of air, air-carbon, carbon- 
hydrogen mixtures, and argon, Pergamon Press, Oxford, 1971.
[8] Y. S. Touloukian et al, Thermophysical Properties of Matter, Volume 10: Thermal Diffusivity, 1973. 